\title{
Changes in Liver Gene Expression Indicate Genetic Pathways Associated with Rabbit Haemorrhagic Disease Infection in Wild Rabbits
}

\author{
Nina Schwensow ${ }^{1}$, Brian Cooke ${ }^{2}$, Joerns Fickel ${ }^{1}$, Walburga Lutz ${ }^{3}$, and Simone Sommer ${ }^{*}, 1$ \\ ${ }^{I}$ Department of Evolutionary Genetics, Leibniz Institute for Zoo and Wildlife Research (IZW), Alfred-Kowalke-Str. 17, \\ 10315 Berlin, Germany \\ ${ }^{2}$ Institute of Applied Ecology, University of Canberra, ACT 2601, Australia \\ ${ }^{3}$ Institute of Wildlife Research, Pützchens Chaussee 228, 53229 Bonn, Germany
}

\begin{abstract}
Rabbit Haemorrhagic Disease Virus (RHDV) is of worldwide significance for both, domestic and wild rabbits (Oryctolagus cuniculus). While the loss of natural populations of wild rabbits in south-western Europe is of major conservation concern because rabbits are a key-stone species in natural ecosystems, the virus has been successfully used as a biological control of pest rabbits in Australia. However, rabbit numbers in Australia are currently rising again suggesting ongoing host adaptive changes. Accordingly, from both conservation and control perspectives it is important to know how rabbits are adapting and to work towards understanding the underlying genetic basis of this adaptation. Pathogenicity of a viral disease is not only influenced by the pathogen itself but likely also by the host's immune gene expression which is poorly understood for wild animals. Here we used cDNA microarrays to obtain a general picture of the genetic pathways expressed in the liver during acute RHD infections in wild rabbits. We found that typical immune response pathways are activated during RHD but also identified differences in our results from those that might have been anticipated from laboratory studies. The down-regulation of cell surface protein genes in wild rabbits that avoided infection also suggests that the lower expression of certain surface proteins could confer protection from RHDV infection. This study expands our understanding of the molecular mechanisms at the host-pathogen interface during RHDV infection and pathogenesis and constitutes a step towards determination of genetic mechanisms that may eventually prove important in host-pathogen co-evolution under natural conditions.
\end{abstract}

Keywords: Gene expression, immune response, microarray, pathway analysis, rabbit haemorrhagic disease, wild rabbits.

\section{INTRODUCTION}

Differences in gene expression levels underlie phenotypic variation of hosts in pathogen resistance [reviewed by 1]. In humans, for instance, gene expression influences the susceptibility to diseases and aberrant gene expression has a significant impact on the pathogenesis of numerous diseases [2-4]. Studies that associate diseases or pathogens with immune gene expression are uncommon for wild animals living in natural conditions [but see 5, 6, 7]. Very little is known about the way in which health and immunity are shaped by coevolving, frequently changing host and parasite genotypes in conjunction with other environmental factors [8]. Indeed, such studies are difficult as extreme variance between individuals is common because factors like age, secondary infections, time point of infection, nutritional status or stress all influence overall gene expression [9]. However, these are the conditions under which natural selection occurs and studies that include this variation potentially add important knowledge on hostparasite co-evolutionary processes. We cannot simply rely on theoretical models and laboratory experiments for advancing our understanding and it is important to confirm

*Address correspondence to this author at the Department of Evolutioary Genetics, Leibniz Institute for Zoo and Wildlife Research (IZW), AlfredKowalke-Str. 17, 10315 Berlin, Germany; Tel: 49305168 313; Fax: +49 30 5126104; E-mail : sommer@izw-berlin.de that suggested evolutionary mechanisms actually take place in the field [8].

The European rabbit (Oryctolagus cuniculus) provides an ideal model for studying gene expression under natural conditions because its genome has already been sequenced, making the application of microarrays straightforward. Two viral diseases, myxomatosis and rabbit haemorrhagic disease (RHD) influence population dynamics of rabbits, although the one currently of greatest interest is RHD, caused by a $(+)$ ss RNA virus classified in the family of Caliciviridae. Since its first report in China in 1984 [10], RHDV spread rapidly to more than 40 countries worldwide. Initial outbreaks resulted in the deaths of millions of free-living and domestic rabbits [11]. Typically, the disease causes fever and sudden death $2-3$ days after natural infection [12]. RHD is of worldwide significance, both as a newly emergent disease affecting the rabbit meat industry and because of its severe impact on wild rabbits with contrasting consequences and applications. In south-western Europe the loss of natural populations of wild rabbits is of major conservation concern, not only for the rabbit itself but also threatening the survival of top predators such as the Iberian lynx (Lynx pardinus), the most endangered cat worldwide [13, 14]. By contrast, the virus has been successfully used as a biological control agent in Australia where rabbits are considered a pest threatening agriculture and natural ecosystems [15]. In recent years, some Australian rabbit populations appear to be less affected 
by RHDV and appear to quicker regain former population sizes after outbreaks [16].

Although naturally selected Australian RHDV field strains are very infective, recent trials suggest that Australian rabbits appear to develop resistance to infection as neither virus nor seroconversion (i.e. the appearance of antibodies against RHDV) could be detected after oral infection with the original biocontrol agent, the Czech RHDV strain 351 (Schwensow and Cooke, unpublished data). Resistance to infection is known from human Caliciviruses (Noroviruses), and in that instance resistance is associated with the expression of certain histo-blood group antigens [17, 18]. A recent report [19] showed evidence for RHDV-straindependent differences in the binding specifity to histo-blood groups (HBG) A, B and $\mathrm{H}$, allowing RHDV strains to preferentially recognize different HBG phenotypes of rabbits. Although no completely resistant phenotypes (as for human Noroviruses) have been found in rabbits there is evidence that individuals that lacked the correct HBG phenotype for the respective RHDV strain were more resistant to lethal infection with a low virus dose while survivors of natural outbreaks showed increased frequency of weakly binding phenotypes for the circulating RHDV strain [19]. Also an increased frequency of certain alleles of Sec-1 genes (which encode enzymes for histo-blood group antigen synthesis) was found among survivors of RHD [20]. These findings indicate that genes belonging to genetic pathways involved in the expression of blood group epitopes might be a target of RHDV driven selection. However, A, B, and $\mathrm{H}$ type antigens are not found on rabbit hepatocytes, although large numbers of viral particles are found in the liver, and liver cells can be infected by RHDV experimentally [21]. The liver is the primary organ of virus replication with early and high virus loads [22-24], but the mechanism by which RHDV infects liver cells is unknown at present. Due to the lack of A, B, and $\mathrm{H}$ type antigens on hepatocytes RHDV can not use histo-blood group antigens as receptors [25] and it is likely that additional mechanisms and genetic pathways contribute for RHD resistance and survival of the disease.

In this study we applied cDNA microarrays to characterize global liver gene expression in wild rabbits. Our aim was to identify pathways involved in the physiological response to RHDV, we therefore compared liver gene expression of rabbits in an acute state of disease with rabbits which had fully recovered past RHD infection and no longer showed clinical signs of disease (seropositive for RHDV antibodies $(\mathrm{AB})=$ survivors $\left.=\mathrm{AB}^{+}\right)$and rabbits that had never been infected (seronegative for RHDV antibodies = never infected $=\mathrm{AB}^{-}$), respectively. Secondly, we aimed to detect potential differences in the level of constitutional immune gene expression between wild rabbits which, from their antibody status, had been infected but had survived RHD and uninfected rabbits that had not been exposed to the virus or had resisted challenge up to the time of capture. For wild-caught rabbits it is impossible to say which of those possibilities applies. However, because all rabbits were caught at the same time, both groups should have had a similar probability of becoming infected making it probable that sero-negative ones had been more resistant to infection. Under the hypothesis that blocking virus entry into cells is a mechanism to prevent or slow infection with RHDV, we predicted differences between RHDV-antibody seronegative rabbits and surviving rabbits in the expression of genes potentially associated with virus entry into the cells. We conducted this study as a step towards the identification of the evolutionary mechanisms during host-pathogen coevolution under natural conditions which are important for the development of resistance to RHD and/or development of protection against an infection with RHDV.

\section{MATERIAL AND METHODS}

Rabbit samples were provided by a federal institute of North Rhine- Westphalia/Germany (Landesbetrieb Wald und Holz NRW, Forschungsstelle für Jagdkunde und Wildschadenverhütung, Bonn, Germany). The rabbits originated from the city of Muenster, federal State of North Rhine-Westphalia. In total we tested the blood of 184 adult rabbits for RHDV-antibody presence by using a competition ELISA, available from the Instituto Zooprofilattico, Brescia, Italy. It is based on the competition between anti-RHDV antibodies adsorbed to a solid phase and those present in the rabbit serum sample. The tracer is a conjugated RHDV monoclonal antibody. Symptom-free but antibody positive individuals $\left(\mathrm{AB}^{+}\right)$were considered to have survived an infection and thus to be resistant to RHD.

Based on the ELISA results we grouped the individuals (represented by RNA samples) into two groups: survivors, which were RHDV-antibody positive rabbits without clinical symptoms (group 'RHDV-AB'), and RHDV-antibody negative rabbits which had never become infected (group 'RHDV-AB') and analysed eight of each group on single microarrays. A third group of rabbits consisted of rabbits acutely and terminally infected with RHDV (group 'acutely infected'). For this group we tested five rabbits that were found dead in rabbit colonies in Muenster, North RhineWestphalia, with the clinical diagnosis of RHD. However, due to progressed decay only one of those had a RNA quality (RIN-value) sufficient for microarray analysis. To increase the number of terminally infected individuals, RNA of five rabbits was provided from a RHDV challenge experiment in Australia (approval for this work was obtained through the University of Canberra Animal Experimentation Ethics Committee approval number CEAE09-06) during which wild caught individuals were orally challenged by a standard dose $\left(1500 \times \mathrm{xD}_{50}\right)$ of RHD virus (Czech strain 351$)$. Individuals infected with RHDV and about to succumb to the disease show a sudden, steep increase in body temperature indicating that death is forthcoming. At that point, the rabbits were killed by cervical dislocation. Liver samples were immediately taken and preserved in RNAlater (Invitrogen, Germany).

All subsequent steps, including RNA isolations, RNA quality assessments, microarray hybridisations and data normalising procedures were performed by the microarray customer service of Miltenyi Biotec, Germany. A commercially available microarray $(4 \mathrm{x} 44 \mathrm{k}$ Whole Rabbit Genome Oligo, Agilent, Germany) was used. The annotation and all subsequent data analyses were performed by the bioinformatics service of Miltenyi Biotec, Germany. Annotation enrichment analyses assign significantly regulated genes (=human Entrez Gene IDs) to biological pathways [see 26 for a review] and were performed using the 
TreeRanker software (Miltenyi Biotec $\mathrm{GmbH}$ ). To assess the enrichment of an annotation, its frequency within a given gene set was compared to that of a background set consisting of all human gene IDs assignable to the probes of the Agilent $4 \times 44 k$ Whole Rabbit Genome Oligo Microarray using Fisher's exact test. This procedure identified groups of significantly regulated genes that are linked in terms of their importance in specific biological functions (e.g. chemokine expression). Details on the used laboratory and analytical techniques are given in the supplemental material S1 'Laboratory and analytical techniques'.

\section{RESULTS}

\section{Regulated Reporters and Mapping to Human Genes}

Out of the 43,600 Agilent reporters of the 4x44k Agilent Whole Rabbit Genome Chip, 11,538 reporters passed all filtering processes and were identified as regulated between the three groups of rabbits ('RHDV-AB', 'RHDV-AB' ' and 'acutely infected') by statistical group comparisons. On the rabbit chip genes are covered by several reporters, thus after mapping to the human genome, non-redundant lists were generated to avoid biases in the subsequent pathway analysis. The non-redundant lists of Entrez gene IDs consisted of a total of 13,192 genes. Of these non-redundant sequences that were found to correspond to human genes, 5,340 were regulated between the rabbit groups.

While only eight genes were identified to be differentially regulated between ' $\mathrm{RHDV}-\mathrm{AB}^{+}$' and 'RHDV$\mathrm{AB}^{-}$individuals, we found large differences when we compared either the group 'RHDV- $\mathrm{AB}^{+}$' or the group 'RHDV-AB' ${ }^{-}$with 'acutely infected' rabbits (Table 1). The vast majority of genes regulated differentially between the groups 'RHDV-AB' ${ }^{+}$and 'acute infected' were similar to those found when groups 'RHDV-AB', and 'acutely infected' were compared (the complete lists of regulated reporters is available as electronic supplemental material S2 'Regulation of reporters'). The pathway analysis narrowed down the high numbers of regulated genes and allowed us to identify potential candidate mechanisms of likely biological importance in RHDV infection.

\section{Comparison of 'RHDV-AB' $v$ s 'Acutely Infected'}

Most annotation enrichment for the genes up-regulated in 'acutely infected' rabbits reflect the effects of the viral infection on the rabbit's immune system and are dominated by a strong general interferon response including both type I interferons and IFN $\gamma$ as also found by Waddell et al. [27] for viral infections in humans. In total, we identified 76 significantly enriched categories in this comparison. The most significant enrichment was observed in genes assigned to the category 'up-regulated after HRV16 (human rhinovirus 16) infection'. Other strongly enriched categories include, among others, 'chemokine production', 'cytokine production', 'apoptosis', 'up-regulated by Wnt pathway', 'antiviral response', 'interleukin (IL) 23-mediated signalling events', 'IL27- mediated signalling events', 'IL12-mediated signalling events', 'IL-6-mediated signalling events', 'cellmarker eosinophils', 'Toll-like receptor signalling pathway' (details and complete list are available as electronic supplemental material S3 'Annotation enrichment analysis results'. The list of down-regulated genes comprises only a few significantly enriched categories. The strongest enrichment was seen for the category 'neurotransmittergated ion channel' which is populated by gammaaminobutyric acid (GABA) receptors and receptors for acetylcholine, glycine, and serotonin. The significance of all other enriched categories including 'keratin-associated protein' and 'melanin biosynthesis' is weaker and most categories contained only few genes.

\section{Comparison of ' $\mathrm{RHDV}-\mathrm{AB}^{+}$, vs 'Acutely Infected' Rabbits}

As in the previous comparison, the identified pathways predominantly reflect the effects of a viral infection on the rabbit's immune system. Thus, the most significant enrichments for the genes up-regulated in 'acutely infected' rabbits compared to ' $\mathrm{RHDV}-\mathrm{AB}^{+}$' rabbits were seen in genes assigned to the categories 'up-regulated after HRV16 (human rhinovirus 16) infection', and by the expression of genes induced by both, type I interferons and IFN $\gamma$ [27]. In this comparison we identified 75 significantly enriched categories. These include 'cellmarkers of any kind of activated immune cells', 'regulations of chemokine production', 'apoptosis', 'IL-23-mediated signalling events', 'Toll-like receptor signalling pathway', 'antiviral defence', 'IL-6-mediated signalling events' and many others (see electronic supplemental material S3 'Annotation enrichment analysis results').

\section{Comparison of 'RHDV-AB', and 'RHDV-AB', Rabbits}

Gene expression between the groups 'RHDV-AB', and ' $\mathrm{RHDV}-\mathrm{AB}^{-}$' was highly similar, both in terms of regulated

Table1. Number of Regulated Genes

\begin{tabular}{|c|c|c|c|}
\hline Comparison & Up-Regulated & Down-Regulated & Total \\
\hline 'RHDV-AB' $v s$ 'RHDV-AB', & 6 & 2 & 8 \\
\hline 'RHDV-AB's $v s$ 'acutely infected' & 1021 & 1773 & 2794 \\
\hline 'RHDV-AB ${ }^{+} v s$ 'acutely infected' & 944 & 1594 & 2538 \\
\hline overlap 'RHDV-AB', and 'RHDV-AB' $v s$ 'acutely infected' & 877 & 1542 & 2419 \\
\hline exclusively regulated in 'RHDV-AB', $v s$ 'acutely infected' & 144 & 231 & 375 \\
\hline exclusively regulated in 'RHDV-AB', $v s$ 'acutely infected' & 67 & 52 & 119 \\
\hline
\end{tabular}

Number of regulated, non-redundant genes, based on human Entrez Gene IDs. "Up"- and "down-regulated" refers to the second group in each comparison, e.g. in the comparison 'RHDV-AB ${ }^{+}$, versus 'RHDV-AB', six genes are more strongly expressed in 'AB', rabbits. Overlap between 'RHDV-AB', and 'RHDV-AB ${ }^{+}$' $v s$ ' acutely infected' shows the number of genes regulated in the same way in RHDV-antibody negative and RHDV-antibody positive rabbits when compared to terminally infected rabbits. 
genes and in the direction of their regulation. This finding was also reflected in the annotation enrichment analysis. From the eight genes that were detected to differ significantly in their expression between these two rabbit groups only two genes were down-regulated in 'RHDV-AB' ' rabbits, thus no enrichment could be tested. However, four out of the six genes up-regulated in ' $\mathrm{AB}^{+}$' rabbits belonged to the category 'surface protein' $(p=0.014$, enrichment factor: 3.54; see electronic supplemental material S3 'Annotation enrichment analysis results').

\section{DISCUSSION}

The understanding of how RHD affects rabbits and what genes and pathways are involved in fighting infection and developing resistance is particularly important for rabbit management, both in areas where they are a pest species threatening the endemic flora and fauna (e.g. Australia), and in areas where disease resistance would aid the recovery of disease-affected populations (e.g. Europe).

Often, microarray analysis is followed by quantitative real-time PCR experiments to confirm the observed expression patterns of certain genes of interest. But at the same time this sacrifices the two main advantages of microarrays: their rapidity and genome wide scope [28]. Our aim was to provide a general picture of the processes important during acute RHD infections to identify potential targets for pathogen-driven selection and not to identify the relative importance of the expression level of single genes. We also aimed to investigate constitutional differences between rabbits that had survived RHD and antibody negative rabbits to narrow down genes potentially involved in resistance to RHDV infection.

\section{Mapping to Human Genes}

Because the rabbit chip (Agilent Whole Rabbit Genome Oligo Microarray) annotation was insufficient, we improved it by mapping the rabbit Agilent reporters to human Entrez gene IDs, which was successful for a third of the reporters. However, for rabbit genes without a clear 'one to one' human ortholog, we cannot exclude the possibility of erroneous assignments. But because this problem mainly affects closely related genes with similar or identical pathway affiliations, the influence on the results of the pathway analysis will likely be neglectable. Due to the fact of having used RNA extracts from liver homogenates we can not determine cell specific (e.g. hepatocytes, Kupffer cells, infiltrating leukocytes) gene expression.

\section{Pathways Involved in Acute RHDV Infection}

\section{Pathways Up-Regulated in 'Acutely Infected' Rabbits}

As expected, the majority of genes up-regulated during RHD reflect general immune reactions to acute viral infection. Such reactions, demonstrated for hepatitis $\mathrm{C}$ virus (HCV) infections in humans [29] comprise inflammatory and adaptive immune responses initiated by host receptors recognising pathogen-associated molecular patterns (PAMP) of the infecting virus. Activated cellular transcription factors then mediate the onset of interferon regulatory factors (IFR) and nuclear factor $\kappa \mathrm{B}(\mathrm{NF}-\kappa \mathrm{B})$, leading to the expression of chemokines and proinflammatory cytokines, such as type I interferons and INF- $\beta$ which in turn activate the Jak-STAT and other pathways [see 29 for a review]. As for HCV the liver is also the first target for RHDV reproduction [22, 30], and although not closely related, both are (+)ssRNA viruses, whose infections lead to inflammatory affection of the liver with consequent liver injury causing multi-organ failure and high mortality. Our data indicate that mechanisms similar to those in HCV infection are important during RHDV infection because for many genes up-regulated in acutely infected rabbits we found significant annotation enrichment for the typical immune response pathways mentioned above. In liver tissue, not only genes associated with interferon type 1 , but also interferon $\gamma$, are up-regulated. During RHDV infections mitogen-activated protein kinase (MAPK) activation occurs in the liver which contributes to the induction of apoptosis [31]. Also NF-kB and AP-1 (activator protein 1) pathways and increased expression of transforming growth factor (TGF) $\beta_{1}$ were found to be induced which is typical in viral infections [31,32]. The same is indicated by our data where similar processes are traceable in unselected, wild rabbits of unknown genetic background and age. It was also suggested that a late or only slightly elevated expression of TGF $\beta_{1}$ may increase survival of RHD as is the case for human liver infections where the expression of TGF $\beta_{1}$ is higher in patients with poor outcome [32 and references therein]. The rabbits investigated in our study had either died from RHDV (one individual) or were terminally infected. Whether a less vigorous response or later expression of $\operatorname{TGF} \beta_{1}$ is a potential resistance mechanism that has evolved under RHDV driven selection in nature would be important to test in future studies.

Our data indicate an interesting difference to results from a recent experimental study which may indicate natural RHDV driven selection. In the experimental study in which white laboratory rabbits were challenged with RHDV there was no activation of genes associated with STAT (signal transducers and activators of transcription) pathways. These genes determine transcription factors involved in the regenerative process during fulminant hepatic failure [31] and blocking the function of STAT proteins may have evolved as a viral mechanism for evading the host's immune defence [31,33]. Interestingly, in our analysis no such blocking was indicated. Australian RHDV-infected wild rabbits showed elevated expression in many genes in the STAT pathway indicating that under natural conditions mechanisms may be evolving to counter this viral evasion strategy.

Apoptosis, another important pathway in RHD pathology [34] is a process of programmed cell death used by organisms to eliminate superfluous, cancerous, or virusinfected cells and a fundamental component of virtually all acute and chronic liver diseases [35]. It is mainly affected by caspases, a family of cysteine proteases which cleave cellular target proteins leading to the destruction of the cell [36]. A high rate of caspase-3 activity was found during experimental RHDV infection [31,37] and in feline calicivirus infections. Interestingly, in the latter virus capsid cleavage was also present. However, whether this is a host cell defence mechanism to interfere with virus assembly or if the cleaved virus protein is involved in pathogenesis [38] is left to future studies. 


\section{Pathways Down-Regulated in 'Acutely Infected' Rabbits}

Only a few annotation-enriched categories were found for the genes down-regulated in infected rabbits. The most significant enrichment was found for genes important in the 'gamma-aminobutyric acid (GABA) signalling pathway' and 'keratin-associated proteins'. This likely reflects liver inflammation or ongoing liver damage. Although GABA is the principal inhibitory amino acid neurotransmitter in the mammalian brain, GABAergic activity is known from many tissues including kidney, skin and liver [39]. The liver possesses specific GABA receptors which are involved in regulating hepatic regenerative activity [40]. Normally, rat and human liver contain high concentration of GABA and its metabolic enzyme systems [41]. However, decreases in GABAergic activity contribute to the pathogenesis of hepatocellular carcinoma [42] and GABA receptor activity is significantly down-regulated in human hepatocellular carcinoma tissues when compared to adjacent, non-tumorous and healthy liver tissues [43]. Higher levels of GABA are protective against the cytotoxicity of ethanol in isolated rat hepatocytes [44]. Like in human hepatocellar carcinomas, GABA pathways also appear to be down-regulated during RHDV infection, presumably associated with increasing liver damage. Whether a higher expression of genes associated with GABA pathways protects liver cells during RHD and if this is a potential mechanism that has evolved under RHDV driven selection needs to be tested.

The down-regulation of keratin and keratin-associated proteins may also reflect the effects of RHDV on the liver. Keratins are the major intermediate filament proteins of the liver and other epithelial tissues [45]. Some keratins are important in modulating the apoptotic response as their absence or mutation increases apoptosis [46-48]. Keratins can act both as targets and modulators of toxic stress and their functional relevance in human diseases has been demonstrated in patients with liver cirrhosis [49]. Keratins are regulated and interact with diverse keratin-associated proteins (KAP) of various functions. Although the roles of some keratin-KAP interactions are not fully understood, especially not in wild animals, some KAPs help maintaining cell mechanical stability, regulate keratin filament organisation. Keratin regulation itself occurs via several modalities including interactions with KAPs [reviewed by 50]. The significant annotation enrichment in downregulated genes of keratin-associated pathways indicates that the decrease of keratins and KAPs may either be a general sign of apoptosis during RHD or may even enhance apoptotic processes. Whether the expression level of keratin or KAP genes influences survival of RHD and may thus play a role in increasing resistance of wild rabbits needs to be investigated.

\section{Constitutional Differences Between RHD-Survivors and Antibody-Negative Rabbits}

Healthy RHDV-antibody negative (RHDV-AB ${ }^{-}$) rabbits were compared with sero-positive animals $\left(\mathrm{RHDV}-\mathrm{AB}^{+}\right.$) to search for evidence for constitutional differences between survivors of RHD and uninfected, potentially resistant rabbits. The background for this was that the expressions of certain HBG antigens and the expression of enzymes important for their synthesis (Sec-1), respectively, had been suggested as a target for RHDV-driven selection in a RHDV strain-dependent manner $[19,20]$. Despite differential binding preferences of RHDV strains to HGB epitopes no evidence for complete protection from infection was observed; all strains tested were deadly infective at higher doses [51]. Our field observations in Australia showed that virtually all live adult rabbits were seropositive, i.e. all had become infected with currently circulating naturally selected RHDV variants and survived the disease. Thus, besides the adaptive expression of specific blood group antigens other immune response genes had contributed to resistance. At least one mechanism that prevents infection seems clear in wild rabbits. Oral infection with a relatively high dose of $\left(150 \times \mathrm{ID}_{50}\right)$ of the Czech strain 351, the biocontrol strain used in Australia, infected only 3 out of 10 rabbits while none of the survivors seroconverted (Cooke and Schwensow, unpublished data). This contrasts older findings, where much lower doses (single flyspot contining 2-3 x $\mathrm{ID}_{50}$ ) easily infected and killed wild rabbits from the same region [52]. Reduced numbers and/or lower expression of virus ligands may have contributed to increased resistance to infection. However, since no HGB antigens are present on hepatocytes $[19,25]$ it is likely that other structures in liver cells serve as ligands for RHDV entry and thus are a potential target of RHDV driven selection.

We chose a broad approach and tested for general, or constitutional, expression differences in liver tissue between rabbits that had survived RHD and seronegative rabbits. Our results do not provide strong evidence for constitutionally different expression between the two groups. Since only two genes were found to be down-regulated in 'RHDV-AB' rabbits when compared to 'RHDV-AB' ones, no enrichment analyses could be performed for this comparison but four of the six genes found to be up-regulated in 'RHDV- $\mathrm{AB}^{+}$, rabbits were associated with 'surface proteins'. However, the genes were not directly functionally linked or had unknown functions, respectively. Although we do not know whether the wild sero-negative adult rabbits were naïve or resistant to infection this suggests that the lower expression of certain surface proteins in the liver of 'RHDV-AB'' rabbits may be important in conferring protection from infection with RHDV. However, to assess the adaptive importance of the regulation of these genes future studies will be needed to decipher their function.

\section{CONCLUSION}

The inclusion of natural variability is very important when studying host-pathogen co-evolution and assessing the health impacts of immune factors and diseases [8]. However, due to lack of sequence information only a few studies to date have characterised gene expression in wild animals and compared immune reactions during infections with results from laboratory studies [but see 5,53]. The analysis of gene expressions presented here comes from wild living rabbits and to our knowledge, this is the first report of the analysis of overall gene expression (more than 43,000 reporters tested on the microarray) of rabbits infected with RHDV. Despite the variance in the samples, as expected for wild animals, we were able to clearly identify genetic pathways that are important during infection with a lethal virus. We found differences in gene expression among wild rabbits from what might be expected from studies on laboratory 
rabbits which may be indicative of natural selection in wild rabbits. Our results are thus a first step in identifying potential areas in immune reactions where host-pathogen coevolutionary processes are likely to take place. Next to adaptation in immune responses our study suggests an additional mechanism likewise to be under virus driven selection. We identified a few surface protein genes that might play a role in either permitting or facilitating virus entry into liver cells. Together with HBGs [19] this might be important for hindering the infection with RHDV or for reducing the severity of the disease. The genetic pathways suggested by our broad approach constitute promising candidates for further studies, preferentially under laboratory conditions. This will be crucial for understanding the RHDV-rabbit co-evolutionary processes in the wild and for identifying the causes for higher current survival rates during natural RHD outbreaks in Australian wild rabbits.

\section{ACKNOWLEDGEMENTS}

The project was funded by the German Science Foundation (DFG) Priority Program 'Host-Parasite Coevolution - Rapid reciprocal adaptation and its genetic basis' (SPP 1399, DFG SO 428/7-1) and the Leibniz-Institute for Zoo and Wildlife Research/Berlin. We thank Gudrun Wibbelt and Claudia Szentiks for pathological investigations and Karin Hönig and John Kowaliski for performing the ELISA tests.

\section{CONFLICT OF INTEREST}

None declared.

\section{REFERENCES}

[1] Whitehead A, Crawford D. Variation in tissue-specific gene expression among natural populations. Genome Biol 2005; 6(2): 13 .

[2] Knight JC. Regulatory polymorphisms underlying complex disease traits. J Mol Med 2005; 83(2): 97-109.

[3] Yan H, Zhou W. Allelic variation in gen expression. Curr Opin Oncol 2004; 16: 39-43.

[4] Kleinjan DA, van Heyningen V. Long-range control of gene expression: emerging mechanisms and disruption in disease. The Am J Human Genet 2005; 76(1): 8-32.

[5] Galindo RC, Ayoubi P, Naranjo V, et al. Gene expression profiles of European wild boar naturally infected with Mycobacterium bovis. Vet Immunol Immunopathol 2009; 129(1-2): 119-25.

[6] Schwensow N, Axtner J, Sommer S. Are associations of immune gene expression, body condition and parasite burden detectable in nature? A case study in an endemic rodent from the Brazilian Atlantic Forest. Infect Genet Evol 2011; 11(1): 23-30.

[7] Galindo RC, Muñoz PM, de Miguel MJ, et al. Gene expression changes in spleens of the wildlife reservoir species, Eurasian wild boar (Sus scrofa), naturally infected with Brucella suis biovar 2. J Genet Genomics 2010; 37(11): 725-36.

[8] Pedersen AB, Babayan SA. Wild immunology. Mol Ecol 2011; 20(5): $872-80$

[9] Apanius V. Stress and immune defense. In: Slater PJB, Rosenblatt JS, Snowdon CT, Milinski M, Eds. Stress and Behavior. San Diego, CA. : Academic Press 1998.

[10] Liu SJ, Xue HP, Pu BQ, Quian NH. A new viral diesase in rabbits. Anim Hus Vet Med 1984; 16: 253-5.

[11] Cooke BD, Fenner F. Rabbit haemorrhagic disease and the biological control of wild rabbits, Oryctolagus cuniculus, in Australia and New Zealand. Wildl Res 2002; 29: 689-706.

[12] Cooke BD. Rabbit haemorrhagic disease: field epidemiology and the managment of wild rabbit populations. Rev Sci Tech 2002; 21(2): 347-58.

[13] Von Arx M, Breitenmoser-Wursten C. Lynx pardinus. In: IUCN 2011. IUCN Red List of Threatened Species Version 2008.
[14] Nowell K. Revision of the Felidae Red List of Threatened Species. In: Authority ISRLF. IUCN/SSC Cat Specialist Group 2002.

[15] Fenner F, Fantini B. Biological control of vertebrate pests: the history of myxomatosis, an experiment in evolution. Wallingford: CABI Publishing 1999.

[16] Mutze G, Bird P, Cooke B, Henzell R. Geographic and seasonal variation in the impact of rabbit haemorrhagic disease on European rabbits, Oryctolagus cuniculus, and rabbit damage in Australia. In: Alves PC, Ferrand N, Hackländer K, Eds. Lagomorph Biology. Heidelberg: Springer Berlin 2008; pp. 279-93.

[17] Le Pendu J, Ruvoën-Clouet N, Kindberg E, Svensson L. Mendelian resistance to human norovirus infections. Semin Immunol 2006; 18(6): 375-86.

[18] Tan M, Jiang X. Norovirus and its histo-blood group antigen receptors: an answer to a historical puzzle. Trends Microbiol 2005; 13(6): 285-93.

[19] Nyström K, Le Gall-Reculé G, Grassi P, et al. Histo-blood group antigens act as attachment factors of rabbit hemorrhagic disease virus infection in a virus strain-dependent manner. PLoS Pathog 2011; 7(8): 1002188 .

[20] Guillon P, Ruvoen-Clouet N, Le Moullac-Vaidye B, et al. Association between expression of the $\mathrm{H}$ histo-blood group antigen, a 1,2fucosyltransferases polymorphism of wild rabbits, and sensitivity to rabbit hemorrhagic disease virus. Glycobiology 2009; 19(1): 21-8.

[21] König M, Thiel H-J, Meyers G. Detection of viral proteins after infection of cultured hepatocytes with rabbit hemorrhagic disease virus. J Virol 1998; 72(5): 4492-7.

[22] Guittré C, Ruvoen-Clouet N, Barraud L, et al. Early stages of rabbit haemorrhagic disease virus infection monitored by polymerase chain reaction. J Vet Med B 1996; 43(1-10): 109-18.

[23] Shien JH, Shieh HK, Lee LH. Experimental infections of rabbits with rabbit haemorrhagic disease virus monitored by polymerase chain reaction. Res Vet Sci 2000; 68(3): 255-9.

[24] Moussa A, Chasey D, Lavazza A, et al. Haemorrhagic disease of lagomorphs: evidence for a calicivirus. Vet Microbiol 1992; 33(14): 375-81.

[25] Ruvoën-Clouet N, Ganière JP, Andrè-Fantaine G, Blanchar D, Le Pendu J. Binding of rabbit hemorrhagic disease virus to antigens of the ABH histo-blood group family. J Virol 2000; 74(24): 11950-4.

[26] Huang DW, Sherman BT, Lempicki RA. Bioinformatics enrichment tools: paths toward the comprehensive functional analysis of large gene lists. Nucleic Acids Res 2009; 37(1): 1-13.

[27] Waddell SJ, Popper SJ, Rubins KH, et al. Dissecting interferoninduced transcriptional programs in human peripheral blood cells. PLoS One 2010; 5(3): 9753.

[28] Kammenga JE, Herman MA, Ouborg NJ, et al. Microarray challenges in ecology. Trends Ecol Evol 2007; 22(5): 273-9.

[29] Gale M, Foy EM. Evasion of intracellular host defence by hepatitis C virus. Nature 2005; 436(7053): 939-45.

[30] Park JH, Ochiai K, Itakura C. Detection of rabbit haemorrhagic disease virus particles in the rabbit liver tissues. J Comp Pathol 1992; 107(3): 329-40.

[31] García-Lastra R, San-Miguel B, Crespo I, et al. Signaling pathways involved in liver injury and regeneration in rabbit hemorrhagic disease, an animal model of virally-induced fulminant hepatic failure. Vet Res 2010; 41(1): 02.

[32] Sânchez-Campos S, Alvarez M, Culebras JM, Gonzalez-Gallego J, Tuñón MJ. Pathogenic molecular mechanisms in an animal model of fulminant hepatic failure: rabbit hemorrhagic viral disease. J Lab Clin Med 2004; 144(4): 215-22.

[33] Duechting A, Tschöpe C, Kaiser H, et al. Human parvovirus B19 NS1 protein modulates inflammatory signaling by activation of STAT3/PIAS3 in human endothelial cells. J Virol 2008; 82(16): 7942-52.

[34] Alonso C, Oviedo JM, Martín-Alonso JM, et al. Programmed cell death in the pathogenesis of rabbit hemorrhagic disease. Arch Virol 1998; 143(2): 321-32.

[35] Schattenberg JM, Galle PR, Schuchmann M. Apoptosis in liver disease. Liver Int 2006; 26(8): 904-11.

[36] Kaufmann SH, Hengartner MO. Programmed cell death: alive and well in the new millennium. Trends Cell Biol 2001; 11(12): 52634.

[37] San-Miguel B, Alvarez M, Culebras J, et al. N-acetyl-cysteine protects liver from apoptotic death in an animal model of fulminant hepatic failure. Apoptosis 2006; 11(11): 1945-57. 
[38] Roberts LO, Al-Molawi N, Carter MJ, Kass GEN. Apoptosis in cultured cells infected with feline calicivirus. Ann N Y Acad Sci 2003; 1010(1): 587-90.

[39] Erdö SL, Wolff JR. $\gamma$-Aminobutyric acid outside the mammalian brain. J Neurochem 1990; 54(2): 363-72.

[40] Erlitzki R, Gong Y, Zhang M, Minuk G. Identification of GAMMAaminobutyric acid receptor subunit types in human and rat liver Am $\mathbf{J}$ Physiol Gastrointest Liver Physiol 2000; 279(4): 733-9.

[41] Minuk GY. Gamma-aminobutyric acid and the liver. Dig Dis 1993; 11(1): 45-54.

[42] Minuk GY. GABA and hepatocellular carcinoma. Mol Cell Biochem 2000; 207(1): 105-8.

[43] Zhang M, Gong Y, Assy N, Minuk GY. Increased GABAergic activity inhibits [alpha]-fetoprotein mRNA expression and the proliferative activity of the HepG2 human hepatocellular carcinoma cell line. J Hepatol 2000; 32(1): 85-91.

[44] Norikura T, Kojima-Yuasa A, Opare Kennedy D, Matsui-Yuasa I. Protective effect of gamma-aminobutyric acid (GABA) against cytotoxicity of ethanol in isolated rat hepatocytes involves modulations in cellular polyamine levels. Amino Acids 2007; 32(3): 419-23.

[45] Omary MB, Ku N-O, Toivola DM. Keratins: Guardians of the liver. Hepatology 2002; 35(2): 251-7.

[46] Caulin C, Ware CF, Magin TM, Oshima RG. Keratin-dependent, epithelial resistance to tumor necrosis factor-induced apoptosis. J Cell Biol 2000; 149(1): 17-22.
[47] Ku N-O, Soetikno RM, Omary MB. Keratin mutation in transgenic mice predisposes to Fas but not TNF-induced apoptosis and massive liver injury. Hepatology 2003; 37(5): 1006-14.

[48] Magin TM, Vijayaraj P, Leube RE. Structural and regulatory functions of keratins. Exp Cell Res 2007; 313 (10): 2021-32.

[49] Zatloukal K, Stumptner C, Fuchsbichler A, et al. The keratin cytoskeleton in liver diseases. J Pathol 2004; 204(4): 367-76.

[50] Coulombe PA, Omary MB. 'Hard' and 'soft' principles defining the structure, function and regulation of keratin intermediate filaments. Curr Opin Cell Biol 2002; 14(1): 110-22.

[51] Nyström K, Grahn A, Lindh M, et al. Virus-induced transcriptional activation of host FUT genes associated with neo-expression of Ley in cytomegalovirus-infected and sialyl-Lex in varicella-zoster virus-infected diploid human cells. Glycobiology 2007; 17(4): 35566.

[52] Asgari S, Hardy JRE, Sinclair RG, Cooke BD. Field evidence for mechanical transmission of rabbit haemorrhagic disease virus (RHDV) by flies (Diptera: Calliphoridae) among wild rabbits in Australia. Virus Res 1998; 54(2): 123-32.

[53] Graham AL, Hayward AD, Watt KA, Pilkington JG, Pemberton JM, Nussey DH. Fitness correlates of heritable variation in antibody responsiveness in a wild mammal. Science 2010; 330(6004): $662-5$.

(C) Schwensow et al.; Licensee Bentham Open.

This is an open access article licensed under the terms of the Creative Commons Attribution Non-Commercial License (http:/creativecommons.org/licenses/by-nc/ $3.0 /$ ) which permits unrestricted, non-commercial use, distribution and reproduction in any medium, provided the work is properly cited. 